\title{
Increasing accuracy and reliability of ground base settlement calculation
}

\author{
Ol'ga Korobova ${ }^{1,{ }^{*}}$, Ljubov Maksimenko ${ }^{2}$, and Dmitrii Grigor'ev ${ }^{1}$ \\ ${ }^{1}$ Novosibirsk State University of Architecture and Civil Engineering (SIBSTRIN), Turgeneva str., \\ 159, 630008, Novosibirsk, Russia \\ ${ }^{2}$ Siberian State University of Geosystems and Technologies, Plakhotnogo str., 10, 630000, \\ Novosibirsk, Russia
}

\begin{abstract}
The article presents the sequence of soil foundations for deformations calculation, taking into account their deformation anisotropy. The calculations were made by the finite element method using the experiment and its mathematical planning. It was found that one can use the numerical experiment results (coefficients tables of soil anisotropy) and calculate soil base sediment and layers of different thickness, using any of the existing methods after determining the index of the soil deformation anisotropy and authenticity, thereby increase their accuracy and reliability.
\end{abstract}

\section{Introduction}

Reliable prediction of the stress-strain state (SSS) of soil foundations is an up-to-date problem of modern construction, which makes it possible to better use the opportunities they possess, i.e. to obtain the most economical options for foundations and structures from the ground while ensuring sufficient safety during their operation, as well as to increase the calculation's accuracy and reliability of soil foundation settlement.

Design models can be considered reliable and economical only if they fully reflect the real properties of soils and the phenomena occurring in the soil under the action of external loads. The available data on the SSS of subsoil grounds basically confirm the provisions of the computational methods, but in some cases there is a need to correct them or even create new calculation methods, including engineering ones. One of the most important issues in the study of SSS is the issue of the deformation anisotropy of soils in the bases calculation.

Regulatory documents for the calculation of ground bases are recommended (SP 22.13330.2016 "Grounds of buildings and structures. The updated edition of SNiP 2.02.01$83 * "$ takes into account the anisotropy of soils, which is often associated with difficulties in the absence of simple and efficient anisotropy, as well as methods for determining design parameters of anisotropic soil.

Meanwhile, numerous studies both in our country and abroad show that all natural nonrock soils have the property of deformation anisotropy, which degree and nature is quite different: they are clearly expressed with a layered or columnar texture (for example, in compacted bulk soils, loess soils, etc.) and less in sandy and clay soils of natural

\footnotetext{
* Corresponding author: michmacha@mail.ru
} 
composition [2-7] and others. Successful solution of this problem is impossible without the use of numerical and mathematical methods in the formation of the computational model; one of the components is the stress-strain state of anisotropic soil study, as well as the development of mechanisms for adjusting the method of sediment foundations calculating.

In this regard, the relevance of the issue under consideration is not subject to doubt. The authors developed a method of anisotropy of the soil deformation, which is as follows. At the first stage, experimental studies are carried out in order to determine the soil anisotropy indicators. First, soil samples are taken in two mutually perpendicular directions (perpendicular and parallel to the bedding of the soil). Then, tests of selected samples will be prolonged under compression conditions, according to the existing standard methods in laboratory conditions. According to the test results, the deformation modules in the vertical and horizontal directions and their relations are calculated (hereinafter, the anisotropy indicators $\alpha$ ).

The degrees evaluation of anisotropy samples deformation was carried out by comparing the values of deformations (modules of deformations) in two mutually perpendicular directions. In order to analyze the obtained results, it is possible to estimate the degree of soils anisotropy deformation by the anisotropy index $\alpha=\mathrm{s}_{\mathrm{x}} / \mathrm{s}_{\mathrm{z}}=\varepsilon_{\mathrm{X}} / \varepsilon_{\mathrm{z}}$; where $\mathrm{s}_{\mathrm{z}}$ and $\mathrm{s}_{\mathrm{x}}, \varepsilon_{\mathrm{z}}$ and $\varepsilon_{\mathrm{x}}$ are absolute and relative deformations in the vertical and horizontal direction, respectively. The anisotropy indicators $\alpha$ of the studied soil types (the total number of tests performed is more than eight hundred samples) were practically shown and varied from 0.5 to 2.1. Sands and clay soils studies expanded the area of soils with the established deformation anisotropy.

Novosibirsk clay soils previously investigated by V.P. Pisanenko [8] was characterized by the values $\alpha=1.43$ (loams) and $\alpha=1.24$ (sandy loams). From the analysis of the obtained results, we can conclude that the nature of anisotropy deformation of the studied soil types is different. The indicator is $\alpha \leq 1$ for plastic sandy loops; for loessy sandy loams, as a rule is $\alpha>1$; for sands of medium density and dense, tested under compression conditions is $\alpha<1$. Within the compressive load increasing, values $\alpha$ increase. The character of anisotropy does not change with an increase in the level of acting stresses under conditions of plane deformation and hydrostatic compression of average density sand. The indicator $\alpha$ is less under conditions of plane deformation, than it is under conditions of hydrostatic reduction.

$\alpha$ indicators sand of average density in these conditions exceed unity, and dense sand is less than unity. The degree of deformation anisotropy can be estimated not only by the ratio of soil samples deformations in orthogonal directions, but also by the ratio $\alpha=E_{z} / E_{x}$ taking into account different values $E_{z}$ and $E_{x}$ of Pousson's ratios (coefficients of soil lateral expansion); $\mathrm{E}_{\mathrm{z}}$ and $\mathrm{E}_{\mathrm{x}}$ are deformation modules in vertical and horizontal directions. By comparing the values of deformations (moduls of deformations), the index of deformation anisotropy is calculated (hereinafter, the anisotropy indicators $\alpha$ ) in two mutually perpendicular directions [9].

For the simplest case of compression sample from a transversely isotropic soil with a horizontal isotropy plane (x y) with the absence of lateral deformations $\boldsymbol{\varepsilon}_{\mathbf{x}}=\boldsymbol{\varepsilon}_{\mathbf{y}}=0$, we consider:

$$
\sigma_{x}=\frac{\sigma_{z} v_{z x}}{\approx\left(1-v_{y x}\right)}
$$

where $\boldsymbol{v}_{\mathbf{z x}}$ and $\boldsymbol{\nu}_{\mathbf{y x}}$ are Poisson's coefficients under the action of stresses $\sigma_{\mathbf{z}}$ and $\sigma_{\mathbf{y}}=\sigma_{\mathbf{x}}$, respectively.

According to vertical deformations $\varepsilon z$, we obtained the following expressions: 


$$
\begin{gathered}
\varepsilon_{z}=\frac{\left[\sigma_{z}\left(1-2 v_{x z} \cdot v_{z x}\right) /\left(1-v_{y x}\right)\right]}{E_{z}} . \\
v_{z x}=v_{x z} \frac{E_{x}}{E_{z}}=\frac{v_{x z}}{\alpha}, \quad \varepsilon_{z}=\frac{\sigma_{z}}{E_{z}} \cdot\left(1-\frac{2 \cdot v_{x z} \cdot v_{z x}}{1-v_{y x}}\right)=\frac{\sigma_{z}}{E_{z}} \cdot\left(1-\frac{2 \cdot v_{x z}}{\alpha \cdot\left(1-v_{y x}\right)}\right)=\frac{\sigma_{z}}{E_{z}} \cdot \beta^{\prime}
\end{gathered}
$$

If $\boldsymbol{\alpha}=1$ и $\boldsymbol{v}_{\mathbf{z x}}=\boldsymbol{v}_{\mathbf{y x}}$, the formula for $\boldsymbol{\varepsilon}_{\mathbf{z}}$ has the transcription, obtained for an isotropic medium. The parameter $E_{x}$ can be found from the condition that the strains $\boldsymbol{\varepsilon}_{\mathbf{x}}$ are zero:

$$
E_{x}=\frac{\sigma_{x} \cdot\left(1-v_{y x}\right)}{\left.\sigma_{z} \cdot v_{z x}\right)} E_{z}=\frac{\xi \cdot\left(1-v_{y x}\right)}{v_{z x}} E_{z},
$$

where $\xi$ is the lateral pressure coefficient of the soil under compression conditions.

So, it is necessary to have five equations relating the $\sigma_{\mathbf{z}}, \sigma_{\mathbf{x}}$ stress measured at compression tests and $\boldsymbol{\varepsilon}_{\mathbf{z}}$ strain (at five levels of the actual load) to determine the five parameters of a transversely isotropic medium. The experiments in the stabilometer are preferable in connection with the difficulty of the stresses $\sigma_{\mathbf{x}}$ determining in the compression device. Thus, V.P. Pisanenko made his researchers [8].

This way is very laborious and represents a separate task. Therefore, we restrict ourselves to simplifying anisotropy assessment methods by the ratio of relative deformations $\varepsilon_{\mathrm{x}}$ and $\varepsilon_{\mathrm{z}}$, equivalently, the ratio of deformation modules $\mathbf{E}_{\mathbf{z}} / \mathbf{E}_{\mathbf{x}}$, calculated from measured $\varepsilon_{\mathrm{z}}$ and $\varepsilon_{\mathrm{x}}$ dependences of the isotropic medium for solving SSS question of an anisotropic base. Thus, the studied soils had deformation modules established by the formulas:

$$
\begin{aligned}
& E_{z}=\beta \cdot \frac{\sigma_{z} \cdot h}{s_{z}} \text { or } E_{z}{ }^{0}=\beta \cdot \frac{\Delta \sigma_{z} \hat{h}}{\Delta s_{z}}, \\
& E_{x}=\beta \frac{\sigma_{x} \|^{k}}{s_{x}} \text { or } E_{x}{ }^{\prime}=\beta \cdot \frac{\Delta \sigma_{x} \cdot h}{\Delta s_{x}} \text {, }
\end{aligned}
$$

where $E_{z}$ and $E_{x}$ are "cross-section" deformation modules of soil samples isolated from an array in horizontal and vertical directions;

$\mathrm{E}_{\mathrm{z}}^{\prime}$ and $\mathrm{E}_{\mathrm{x}}^{\prime}$ are the same, "step-by-step" deformation modules;

$\beta=1-\frac{2 v^{2}}{1-v}-$ coefficient of transverse soil expansion absence in a compression device; $\boldsymbol{v}$ - a lateral expansion coefficient; $\mathrm{h}=0.035$ - a ring height.

The modules of soil deformation determination were performed under the action of compaction pressures $\sigma_{\mathrm{z}}$ on samples ranging from 0.05 to $0.30 \mathrm{MPa}$. At loading each step, the "secant" and "stepping" deformations modules of various tested soils in orthogonal directions were calculated.

The "secant" deformation modules were calculated at each subsequent loading stage (with a difference in readings between 0 and subsequent readings); "Step" on the difference between the steps (with the differences between the next and previous steps). 
Table 1. Statistical indicators of the "crossing" modulus of deformation $\mathrm{E}_{z}$.

\begin{tabular}{|l|c|c|c|c|c|c|}
\hline \multirow{2}{*}{ Test results } & \multicolumn{7}{|c|}{ The value of sealing pressure, $\sigma_{\mathrm{z}}, \mathrm{MPa}$} \\
\cline { 2 - 7 } & 0.05 & 0.10 & 0.15 & 0.20 & 0.25 & 0.30 \\
\hline The number of test samples, $\mathrm{n}$. & 6 & 6 & 6 & 6 & 6 & 6 \\
\hline $\begin{array}{l}\mathrm{E}_{\mathrm{z}}, \mathrm{MPa} \text { Arithmetic average, } \mathrm{E}_{\mathrm{z}}, \\
\mathrm{MPa}\end{array}$ & 2.3 & 2.8 & 2.9 & 2.9 & 2.9 & 2.9 \\
\hline Standard deviation $\pm \sigma, \mathrm{MPa}$ & 0.4 & 0.2 & 0.3 & 0.2 & 0.4 & 0.2 \\
\hline $\begin{array}{l}\text { Arithmetic average error, } \mathrm{m}, \\
\mathrm{MPa}\end{array}$ & 1.6 & 0.9 & 1.4 & 1.0 & 1.6 & 1.0 \\
\hline Accuracy rate,P, $\%$ & 7 & 3 & 5 & 3 & 6 & 3 \\
\hline Variation coefficient, $\mathrm{V}_{\mathrm{E}}, \%$ & 17 & 8 & 12 & 8 & 14 & 8 \\
\hline
\end{tabular}

Table 2. Statistical indicators "step" modulus of deformation $\mathrm{E}_{\mathrm{z}}^{\prime}$.

\begin{tabular}{|l|c|c|c|c|c|c|}
\hline \multirow{2}{*}{ Test results } & \multicolumn{7}{|c|}{ The value of sealing pressure, $\sigma_{\mathrm{z}}, \mathrm{MPa}$} \\
\cline { 2 - 7 } & 0.05 & 0.10 & 0.15 & 0.20 & 0.25 & 0.30 \\
\hline The number of test samples, $\mathrm{n}$. & 6 & 6 & 6 & 6 & 6 & 6 \\
\hline $\begin{array}{l}\text { Ez, MPa Arithmetic average, } \\
\text { Ez, MPa }\end{array}$ & 2.3 & 7.7 & 9.2 & 12.0 & 15.3 & 20.4 \\
\hline Standard deviation $\pm \sigma, \mathrm{MPa}$ & 0.4 & 0.4 & 0.6 & 0.6 & 0.8 & 1.2 \\
\hline $\begin{array}{l}\text { Arithmetic average error, m, } \\
\mathrm{MPa}\end{array}$ & 1.6 & 1.7 & 2.3 & 2.5 & 3.3 & 5.1 \\
\hline Accuracy rate, $\mathrm{P}_{\mathrm{E}}, \%$ & 7 & 2 & 3 & 2 & 2 & 3 \\
\hline Variation coefficient, $\mathrm{V}_{\mathrm{E}}, \%$ & 17 & 6 & 6 & 5 & 5 & 6 \\
\hline
\end{tabular}

SSS experimental and numerical studies of anisotropic soil bases, carried out on a computer (on various software systems based on the ideas of the finite element method) confirmed the deformation anisotropy of soils. The calculations considered a model of a continuous, linearly deformable, homogeneous, anisotropic medium with a transversely isotropic anisotropy character, described by the parameters $E_{x}, E_{z}, v_{z x}, v_{y x}, G_{x z}$.

Further, numerical studies of the anisotropic soil bases [10] on computers (on various software systems based on the ideas of the finite element method) were carried out using the mathematical planning of the experiment. In this case, a model of a continuous, linearly deformable, homogeneous, anisotropic medium with a transversely isotropic anisotropy was considered. An anisotropic soil base model was used in the calculations, described by the parameters $E_{x}, E_{z}, v_{z x}, v_{y x}, G_{x z}$.

\section{Materials and Methods}

Influence of each anisotropy parameter on the response (stress) functions was evaluated [10], applying the methodology of the experiment's mathematical planning. The factors varying on three levels were Ex, Ez, vzx and vyx. The levels and interval of variation are given in Table 3.

As a result of numerical studies, the stresses indicators $\sigma z \alpha, \sigma x \alpha$ and $\tau$ zx $\alpha$ for an anisotropic medium and the corresponding $\sigma \mathrm{z}, \sigma \mathrm{x}$ and $\tau \mathrm{zx}$ stresses of the isotropic variant $(\alpha=1.0)$ were obtained. The authors developed a method that takes into account the deformation anisotropy of the base soils using the coefficients of the effect of the soil anisotropy, comparing the calculating results of uniformly anisotropic SSS and isotropic bases in the form of layers of different thickness and half-plane. 
Table 3. Parameters variation of the medium deformation anisotropy.

\begin{tabular}{|c|c|c|c|c|c|}
\hline \multirow{2}{*}{ Variation factors } & \multirow{2}{*}{$\mathrm{x}_{\mathrm{i}}$} & \multicolumn{3}{|c|}{ Levels of variation } & Variat \\
\cline { 3 - 5 } & & lower & zero & Upper & interv \\
\cline { 3 - 5 } & & -1 & 0 & 1 & al \\
\cline { 3 - 5 } & $\mathrm{x}_{1}$ & 5 & 22.5 & 40 & 17.5 \\
\hline $\mathrm{E}_{\mathrm{z}}, \mathrm{MPa}$ & $\mathrm{x}_{2}$ & 5 & 22.5 & 40 & 17.5 \\
\hline $\mathrm{E}_{\mathrm{x}}, \mathrm{MPa}$ & $\mathrm{x}_{3}$ & 0.25 & 0.30 & 0.35 & 0.05 \\
\hline$v_{\mathrm{zx}}$ & $\mathrm{x}_{4}$ & 0.25 & 0.30 & 0.35 & 0.05 \\
\hline$v_{\mathrm{yx}}$ & & \multicolumn{4}{|c|}{}
\end{tabular}

A method was developed that takes into account the deformation anisotropy of base soils using the coefficients of soil anisotropy [11] comparing the calculation results of the stress-strain state of uniformly anisotropic and isotropic bases in the form of different thickness and half-plane layers.

These coefficients showed what proportion of the stress in an isotropic medium was the corresponding stress in the anisotropic one. The values of the coefficients are calculated by the formulas:

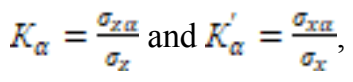

where $K_{\alpha}$ and $K_{\alpha}{ }^{\prime}$ are the correction coefficients of the soil anisotropy influence; $\sigma_{z \alpha}$ and $\sigma_{\mathrm{x} \alpha}$ are vertical and horizontal stresses for an anisotropic medium; $\sigma_{\mathrm{z}}$ and $\sigma_{\mathrm{x}}$ are the same for isotropic.

The stresses $\sigma_{z \alpha}$ and $\sigma_{\mathrm{x} \alpha}, \sigma_{\mathrm{z}}$ and $\sigma_{\mathrm{x}}$ are calculated for characteristic points of the soil massif, located on the central and angular verticals of the loaded surface area, in accordance with the requirements [1]. Correction factors of soil anisotropy effect can be used to adjust the values of sediment foundations, calculated by any of the currently existing methods [1]. The data obtained are sufficient for calculating the sediment of foundations located on the surface of a half-plane or different thickness layers.

The coefficients $K_{\alpha}$ and $K_{\alpha}{ }^{\prime}$ are calculated for the midpoints of the horizontal layers assigned under the base of the foundation according to [1] through $0.4 b$ ( $b$ is the width of the loaded base portion equal to the width of the basement).

When this voltage is calculated by dependencies:

$$
\begin{aligned}
& \sigma_{z p i a}=\sigma_{z p \mathbb{i}^{*}} K_{\alpha}
\end{aligned}
$$

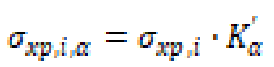

$$
\begin{aligned}
& \sigma_{\text {zpiac }}^{y}=\sigma_{\text {zpi }}^{y} \cdot K_{\mathrm{c}}^{y}
\end{aligned}
$$

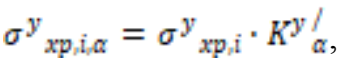

where $\quad \sigma_{z p}\left(\sigma^{y}{ }_{z p}\right)$ and $\sigma_{x p}\left(\sigma^{y}{ }_{x p}\right)$ at the corresponding isotropic points

half-planes are determined by the available solutions for a linearly deformable medium (for example, [1]);

$K_{\alpha}\left(K^{y}{ }_{\alpha}\right)$ и $K_{\sigma}^{\prime}\left(K^{y /}{ }_{\alpha}\right)$ - soil anisotropy influence coefficients for characteristic points of the central and angular verticals.

The values are found by interpolating the results if it is necessary to determine $K_{\alpha}\left(K^{y}{ }_{\alpha}\right)$ and $K_{\sigma}^{v}\left(K^{y /} \sigma_{\sigma}\right)$ values for other points of the central and angular verticals. The values $K_{\alpha}\left(K^{y}{ }_{\alpha}\right)$ and $K_{\alpha}\left(K^{y /}{ }_{\alpha}\right)$ are obtained by comparing the corresponding stresses calculated by the finite element method for $\alpha=1$ and $\alpha \neq 1$ 


\section{Results}

As an example, Table 4 shows the values of the correction factors $\mathrm{K}_{\alpha}$, calculated for the central vertical with anisotropy indices from $\alpha=0.22$ to $\alpha=4.5$ and different layer powers from $h \mid b=1.25$ to the half-plane.

Table 4. Values of correction factors at stress $\sigma_{z}$, central vertical (by-load).

\begin{tabular}{|c|c|c|c|c|c|c|c|c|c|c|c|c|c|c|c|c|}
\hline \multicolumn{17}{|c|}{ Voltage $\sigma_{z}$, central vertical (band load) } \\
\hline \multicolumn{17}{|c|}{ correction factors $\alpha=0.22$ SIGMA 1 Vertical 1} \\
\hline \multirow{2}{*}{$\begin{array}{c}\text { relative } \\
\text { depth }\end{array}$} & \multicolumn{16}{|c|}{ the values of the correction factors were estimated for: } \\
\hline & $0.2 \mathrm{~b}$ & $0.6 \mathrm{~b}$ & $1.0 \mathrm{~b}$ & $1.4 \mathrm{~b}$ & $1.8 \mathrm{~b}$ & $2.2 b$ & $2.6 \mathrm{~b}$ & $3.0 \mathrm{~b}$ & $3.4 \mathrm{~b}$ & $3.8 \mathrm{~b}$ & $4.2 \mathrm{~b}$ & $4.6 \mathrm{~b}$ & $5.0 \mathrm{~b}$ & $5.4 \mathrm{~b}$ & $5.8 \mathrm{~b}$ & $6.2 \mathrm{~b}$ \\
\hline $1-250$ & 0.9 & 0.75 & 0.66 & 0 & 0 & 0 & 0 & 0 & 0 & 0 & 0 & 0 & 0 & 0 & 0 & 0 \\
\hline $2-500$ & 0.9 & 0.76 & 0.66 & 0.6 & 0.57 & 0.55 & 0 & 0 & 0 & 0 & 0 & 0 & 0 & 0 & 0 & 0 \\
\hline $3-750$ & 0.9 & 0.77 & 0.67 & 0.62 & 0.58 & 0.56 & 0.55 & 0.53 & 0.52 & 0 & 0 & 0 & 0 & 0 & 0 & 0 \\
\hline $4-700$ & 0.9 & 0.78 & 0.68 & 0.62 & 0.6 & 0.58 & 0.58 & 0.58 & 0.58 & 0.58 & 0.58 & 0.58 & 0 & 0 & 0 & 0 \\
\hline $6-560$ & 0.9 & 0.78 & 68 & \begin{tabular}{|l|}
0.63 \\
\end{tabular} & 0.61 & 0.6 & 0.61 & 0.62 & 0.64 & 0.66 & \begin{tabular}{|l|}
0.68 \\
\end{tabular} & 0.7 & 0.72 & 0.75 & 0.77 & \begin{tabular}{|l|}
0.77 \\
\end{tabular} \\
\hline \multicolumn{17}{|c|}{ correction factors $\alpha=0.562$ SIGMA 1 Vertical 1} \\
\hline \multirow{2}{*}{$\begin{array}{l}\text { relative } \\
\text { depth }\end{array}$} & \multicolumn{16}{|c|}{ the values of the correction factors were estimated for: } \\
\hline & $0.2 b$ & $0.6 b$ & $1.0 \mathrm{~b}$ & $1.4 \mathrm{~b}$ & $1.8 \mathrm{~b}$ & $2.2 b$ & $2.6 \mathrm{~b}$ & $3.0 \mathrm{~b}$ & $3.4 \mathrm{~b}$ & $3.8 \mathrm{~b}$ & $4.2 b$ & $4.6 \mathrm{~b}$ & $5.0 \mathrm{~b}$ & $5.4 \mathrm{~b}$ & $5.8 \mathrm{~b}$ & $6.2 b$ \\
\hline $1-250$ & 0.97 & 0.93 & 0.89 & 0 & 0 & 0 & 0 & 0 & 0 & 0 & 0 & 0 & 0 & 0 & 0 & 0 \\
\hline $2-500$ & 0.97 & 0.93 & 0.89 & 0.86 & 0.85 & 0.84 & 0 & 0 & 0 & 0 & 0 & 0 & 0 & 0 & 0 & 0 \\
\hline $3-750$ & 0.97 & 0.93 & 0.89 & 0.86 & 0.85 & 0.84 & 0.82 & 0.82 & 0.81 & 0 & 0 & 0 & 0 & 0 & 0 & 0 \\
\hline $4-700$ & 0.97 & 0.93 & 0.89 & 0.86 & 0.85 & 0.84 & 0.84 & 0.83 & 0.82 & 0.82 & 0.82 & 0.82 & 0 & 0 & 0 & 0 \\
\hline $6-560$ & 0.97 & 0.93 & 0.89 & 0.87 & 0.86 & 0.85 & 0.85 & 0.85 & 0.85 & 0.86 & 0.86 & 0.86 & 0.86 & 0.87 & 0.88 & 0.88 \\
\hline \multicolumn{17}{|c|}{ correction factors $\alpha=.778$} \\
\hline relative & \multicolumn{16}{|c|}{ the values of the correction factors were estimated for: } \\
\hline & $0.2 \mathrm{~b}$ & $0.6 \mathrm{~b}$ & $1.0 \mathrm{~b}$ & $1.4 \mathrm{~b}$ & $1.8 \mathrm{~b}$ & $2.2 \mathrm{~b}$ & $2.6 \mathrm{~b}$ & $3.0 \mathrm{~b}$ & $3.4 \mathrm{~b}$ & $3.8 b$ & $4.2 \mathrm{~b}$ & $4.6 \mathrm{~b}$ & $5.0 \mathrm{~b}$ & $5.4 \mathrm{~b}$ & $5.8 \mathrm{~b}$ & $6.2 b$ \\
\hline $1-250$ & 1.02 & 1.06 & 1.09 & 0 & 0 & 0 & 0 & 0 & 0 & 0 & 0 & 0 & 0 & 0 & 0 & 0 \\
\hline $2-500$ & 1.02 & 1.05 & 1.09 & 1.11 & 1.13 & 1.14 & 0 & 0 & 0 & 0 & 0 & 0 & 0 & 0 & 0 & 0 \\
\hline $3-750$ & 1.02 & 1.06 & 1.09 & 1.11 & 1.13 & 1.14 & 1.15 & 1.16 & 1.16 & 0 & 0 & 0 & 0 & 0 & 0 & 0 \\
\hline $4-700$ & 1.02 & 1.05 & 1.09 & 1.11 & 1.13 & 1.14 & 1.15 & 1.16 & 1.16 & 1.17 & 1.17 & 1.17 & 0 & 0 & 0 & 0 \\
\hline $6-560$ & 1.02 & 1.06 & 1.09 & 1.11 & 1.13 & 1.14 & 1.14 & 1.15 & 1.15 & 1.14 & 1.15 & 1.14 & 1.13 & 1.14 & 1.13 & 1.13 \\
\hline \multicolumn{17}{|c|}{ correction factors $\alpha=4.500$} \\
\hline relative & \multicolumn{16}{|c|}{ the values of the correction factors were estimated for: } \\
\hline & $0.2 \mathrm{~b}$ & $0.6 \mathrm{~b}$ & $1.0 \mathrm{~b}$ & $1.4 \mathrm{~b}$ & $1.8 \mathrm{~b}$ & $2.2 b$ & $2.6 \mathrm{~b}$ & $3.0 \mathrm{~b}$ & $3.4 \mathrm{~b}$ & $3.8 \mathrm{~b}$ & $4.2 b$ & $4.6 \mathrm{~b}$ & $5.0 \mathrm{~b}$ & $5.4 \mathrm{~b}$ & $5.8 \mathrm{~b}$ & $6.2 b$ \\
\hline $1-250$ & 1.04 & 1.12 & 1.21 & 0 & 0 & 0 & 0 & 0 & 0 & 0 & 0 & 0 & 0 & 0 & 0 & 0 \\
\hline $2-500$ & 1.04 & 1.12 & 1.21 & 1.28 & 1.32 & 1.35 & 0 & 0 & 0 & 0 & 0 & 0 & 0 & 0 & 0 & 0 \\
\hline $3-750$ & 1.04 & 1.12 & 1.21 & 1.28 & 1.32 & 1.35 & 1.37 & 1.38 & 1.39 & 0 & 0 & 0 & 0 & 0 & 0 & 0 \\
\hline $4-700$ & 1.04 & 1.12 & 1.2 & 1.27 & 1.31 & 1.34 & 1.36 & \begin{tabular}{|l|}
1.37 \\
\end{tabular} & 1.38 & 1.38 & 1.38 & 1.37 & 0 & 0 & 0 & 0 \\
\hline $6-560$ & 1.04 & 1.12 & 1.2 & 1.27 & 1.31 & 1.34 & 1.35 & 1.36 & 1.37 & 1.37 & 1.37 & 1.36 & 1.35 & 1.36 & 1.36 & 1.35 \\
\hline
\end{tabular}

An improved practical method of accounting for deformation anisotropy can be recommended for introduction into practice of designing foundations for buildings under construction, reconstruction and restoration, as well as for the buildings and structures construction in difficult ground conditions. The coefficients determined for the angular vertical points are necessary for calculating the sediment taking into account the effect of 
loads from the neighboring foundations. The values of horizontal stresses $\sigma \mathrm{x}$ and $\sigma \mathrm{x} \alpha$ are used to calculate the sediment by more rigorous methods, which take into account the possibility of base soil's lateral deformations.

Precipitation determination; used in the design of strip foundations for local soil conditions ("KGS" LLP, Kazakhstan); used in the calculation of sediment strip foundations of several industrial and administrative buildings of the Institution UF-91/2 (Novosibirsk), as well as in the calculation of tape foundation precipitation under the reconstructed residential five-story building commissioned by the Federal State Unitary Enterprise - Altai Trust of engineering and construction surveys "(FSUE" Al-TITIS ").

The study results are used in special courses lecturing, elective courses, preparing coursework, degree projects and dissertations for students of "Unique Buildings and Structures Construction", "Industrial and Civil Engineering" and "Urban Construction and Economy" specialties.

\section{Conclusions}

Thus, the proposed improved practical method for calculating soil bases by deformations makes it possible to calculate the settlement of foundations accurately and reasonably, taking into account their real properties [11, 12]. The developed tables of correction coefficients of the soil anisotropy effect can be recommended for calculating the sediment of foundations taking into account the deformation anisotropy according to SP 22.13330.2016 "Foundations of buildings and structures.

\section{References}

1. G.M. Beach, A.A. Kushnireva, Construction and architecture, Novosibirsk 4, 159-161 (1973)

2. A.K. Bugrov, A.I. Golubev, Anisotropic soils and foundation structures (Nedra, SPb, 1993)

3. P.D. Vulis, PhD Thesis (Sverdlovsk, 1973)

4. M.N. Goldstein, V.B. Lapkin, Questions of geotechnics 21, 68-85 (1972)

5. V.A. Kuzmitsky, High School 2, 243-251 (1973)

6. O.A. Korobova, Engineering surveys 6, 24-32 (2012)

7. O.A. Korobova, PhD Thesis (Altai State Technical University, Barnaul, 2002)

8. A.P. Krivorotov, O.A. Korobova, Influence of the deformation anisotropy of the soil on the precipitation of hard foundations (Novosibirsk, 1987)

9. O.A. Korobova, Interexpo Geo-Siberia, Coll. materials XI Intern. scientific conf, Novosibirsk, SSUHT 1, 194 - 199 (2015) 